\title{
Control of single emitter radiation by polarization- and position-dependent activation of dark antenna modes
}

\author{
Mikolaj Kajetan Schmidt, ${ }^{1, *}$ Sebastian Mackowski, ${ }^{2}$ and Javier Aizpurua ${ }^{1}$ \\ ${ }^{1}$ Donostia International Physics Center DIPC and Centro de Física de Materiales CSIC-UPV/EHU, \\ Paseo Manuel de Lardizabal, 5, Donostia-San Sebastián, 20018, Spain \\ ${ }^{2}$ Institute of Physics, Nicolaus Copernicus University, Grudziadzka 5/7, 87-100 Torun, Poland \\ *Corresponding author: mikolaj_schmidt@ehu.es
}

Received December 12, 2011; accepted January 14, 2012;

posted January 30, 2012 (Doc. ID 159789); published March 7, 2012

\begin{abstract}
We study the modification of the decay rates of a single dipolar emitter positioned in the vicinity of metallic linear nanoantennas when higher-order plasmonic excitations are induced. We show that it is possible to effectively tune the enhancement or suppression of both the radiative and nonradiative decay processes by controlling the position and orientation of the dipole with respect to the antenna. Transverse polarization of a single emitter, with respect to the antenna axis, located at the center of the antenna activates dark antenna modes that modify dramatically both the intensity and the spectral features of the decay rates. (c) 2012 Optical Society of America

OCIS codes: $350.5610,350.4238,250.5403$.
\end{abstract}

Small metallic particles, utilized in various branches of nano-optics $[\underline{1}, 2]$ as optical antennas, are capable of localizing the energy of incident radiation into volumes of sizes far below the wavelength of incoming waves [1]. By reciprocity, antennas also serve as radiation broadcasting systems that tune and shape the emission from a localized energy source such as a single dipolar emitter. Numerous designs of metallic structures have already been proposed, realized, and thoroughly investigated $[\underline{1}, \underline{3}, \underline{4}]$. In the majority of these designs, the enhancement of radiation from an emitter is mediated by the excitation of the elementary "bright" dipolar modes of plasmon excitations, which couple efficiently to the outgoing radiation. However, the so-called dark modes of metallic antennas have recently been proposed as a mechanism to introduce new elements of control in the emission of a single emitter [ $[\underline{5}, \underline{6}]$. In particular, it has been reported by Liu et al. [5] that a localized electric dipole positioned in the vicinity of a bipyramidal gold nanoparticle can efficiently excite higher-order multipolar plasmon modes. We elaborate by analyzing the excitation of higher-order modes in linear antennas that sustain dark modes and show how these modes can be used to tune the spectral and radiative properties of a single dipolar emitter located nearby.

In this letter, we consider a simple system, comprising a gold nanorod acting as an antenna and a localized dipolar emitter, as an elementary local feed. The coupling between the dipolar emitter and the oscillations of the free electron gas in the metal is mediated by the electric field originated by the local emitter. Longitudinal and transverse orientations of the emitter with respect to the antenna induce plasmon modes, which exhibit different symmetries. We are interested in two of the specific orientations of the dipole, placed in the $x z$ plane as depicted in the schematics of Fig. 1. An emitter aligned perpendicularly to this plane (along the $y$ axis) induces a weak inhomogeneity of the field in the antenna and exhibits somewhat intermediate results.

Full electrodynamical calculations of electric fields in our antenna-feed system have been carried out with the use of the boundary element method (BEM) [7] to solve
Maxwell's equations. The dielectric function of gold is taken from the literature [8]. The medium surrounding the system is characterized by the dielectric constant of vacuum equal to 1 . The emitter is considered to be a classical electric point dipole with no intrinsic losses, emitting at wavelength $\lambda_{0}$. We use common definitions of radiative decay rates: $\Gamma_{0}$ and $\Gamma_{r}$ for the dipole isolated and in the presence of the antenna, respectively. The rate of nonradiative processes within the system is denoted as $\Gamma_{n r}$. Radiative rates are calculated by integrating the flow of the Poynting vector through a large sphere enclosing the antenna and emitter, while the total decay rate $\Gamma_{t}=$ $\Gamma_{r}+\Gamma_{n r}$ is obtained by examining Green's function of the emitter evaluated at the position of the emitter [9]. Since we are interested mainly in the relative rates of the aforementioned processes, we will further consider

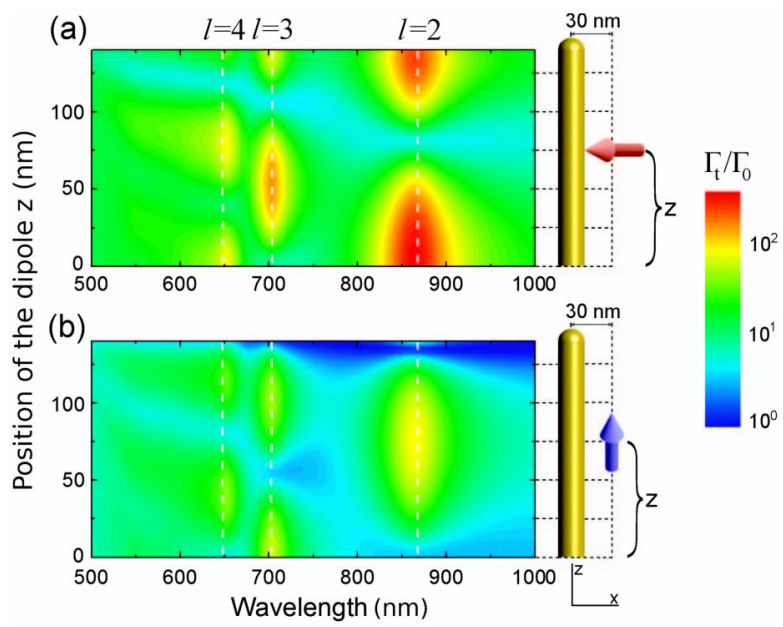

Fig. 1. Enhancement of the total decay rate of an emitter as a function of the radiation wavelength and the space position of the dipole along the antenna axis, at the lateral distance of $30 \mathrm{~nm}$ from the antenna axis. Calculations were performed for the emitter aligned perpendicularly to the antenna axis (upper plot) and parallelly (lower plot). The longitudinal position of the emitter ( $z$ axis) is shown for half of the antenna from its center. Dashed lines indicate the positions of the longitudinal plasmonic antenna resonances. 
the enhancement of the radiative decay given by the ratio $\Gamma_{r} / \Gamma_{0}$ and of the total decay given by $\Gamma_{t} / \Gamma_{0}$.

We first consider a system where the dipolar emitter is positioned in the $x z$ plane at a distance of $30 \mathrm{~nm}$ from the antenna axis and shifted along the length of the gold nanorod from the center of the rod, as depicted in Fig. 1. Metallic nanorods with high aspect ratio-a common choice for linear nanoantennas-exhibit a dipolar longitudinal resonance at a wavelength linearly dependent on the total length [10] (this behavior can be extended to higher-order modes, as we will show below). In our case, a nanorod of total length $300 \mathrm{~nm}$ and $20 \mathrm{~nm}$ diameter is terminated with hemispherical caps of $10 \mathrm{~nm}$ radius.

The enhancement of the total decay rate is plotted in Fig. 1 as a function of the position of the emitter along the nanoantenna and the wavelength of radiation emitted. The antenna resonance wavelengths have been marked with dashed lines for further identification.

The maps of decay in Fig. 1 show maxima at the plasmon resonance wavelengths marked with dashed lines $(l=2,3,4)$ and exhibit no spectral shift between the dipole orientations. As observed in Fig. 1 , these excitations are strongly dependent on the emitter's orientation, meaning that it is possible to trigger the decay rate of the dipole by either switching its orientation or by shifting the antenna with respect to the emitter. For the lowest energy resonance shown in Fig. $1(l=2)$, shifting of the transverse dipole [Fig. 1(a)] from $z=0$ to $80 \mathrm{~nm}-$ by about a quarter of the antenna length-enhances the total decay rate by almost 2 orders of magnitude. Even smaller shifts at the wavelengths corresponding to the higher-energy modes $(l=3,4)$ can trigger considerable changes in the decay rates.

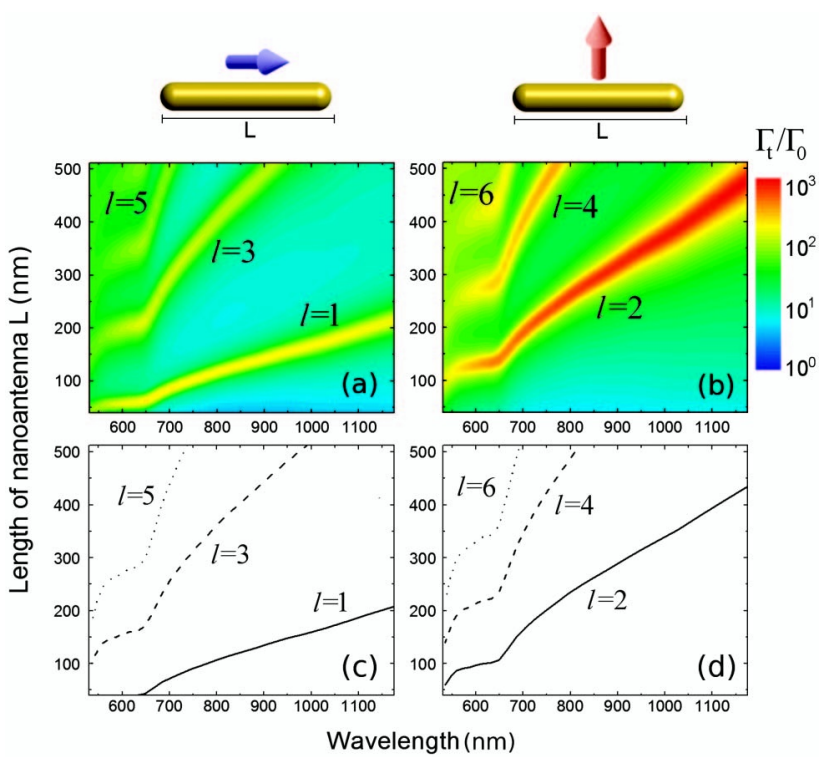

Fig. 2. Antenna modes excitation from (a), (b) full electrodynamic calculations and (c), (d) the effective wavelength theory. In the top row, the dependence of the total decay rate enhancement on the radiation wavelength and the size of the nanoantenna is shown, as obtained from numerical simulations. The emitter is positioned near the middle of the antenna, $30 \mathrm{~nm}$ from its axis and aligned either (a) longitudinally or (b) transversely to the antenna. The width of the nanoantenna is set to $20 \mathrm{~nm}$.
Results shown in Fig. 1 suggest that the plasmon resonances in nanorods have a longitudinal character. It is well known that the correspondence between the length of a linear nanoantenna $L$ and its longitudinal dipolar mode wavelength is given by $L=\lambda_{\text {eff }} / 2$ [10], where $\lambda_{\text {eff }}$ is the plasmon wavelength along the antenna. With the elementary dipolar $l=1$ mode extensively investigated $[11,12]$, we attempt to verify the validity of this effective wavelength theory for higher-order antenna modes $(l=2,3,4, \ldots)[\underline{13}]$, for which the optimal antenna length is given by $L=\overline{l \lambda}_{\text {eff }} / 2$.

We compare the results of electrodynamical BEM calculations with the effective wavelength theory, by plotting the dependence of the first few lowest-order longitudinal resonances on the length of the antenna. For BEM simulations, we have placed the dipolar emitter near the middle of the antenna and oriented it either parallelly or perpendicularly. Maps of total decay rate enhancement are shown in the top row of Fig. 2. The orientation of the dipoles is shown in the schematics on the top of the figure.

Optimal antenna lengths as obtained from the effective wavelength theory [10], with a correction owing to an additional phase shift at the end caps, are shown in the lower row of Fig. 2. Theoretical predictions [Figs. 2(c) and 2(d)] almost perfectly match the results of numerical calculations [Figs. 2(a) and 2(b)], thus validating the applicability of effective wavelength theory to higher-order modes and confirming the nature of resonances as higher-order longitudinal modes.

In Fig. 2 we have separated the results of effective wavelength theory by the parity of the mode number $l$. A comparison to the results of numerical simulations shows that when the dipole is positioned near the middle of the nanoantenna, it selectively couples to even (for transverse orientation) or odd (for longitudinal orientation) $l$ modes. Note that the parity of $l$ determines the net dipole moment of the mode, with even $l$ resonances having a vanishing and odd $l$ a nonvanishing net dipole moment, respectively. Therefore, even and odd $l$ modes can be identified as "dark" and "bright" plasmon antenna modes, respectively.

To get more detailed insights into the mechanism of orientational selectivity of the excitations, we show in Fig. 3 the spectra of the radiative and total decay rate enhancements for a dipolar emitter positioned at the middle of a $300 \mathrm{~nm}$ long nanoantenna. The plots in Figs. 3(a) and 3(b) correspond to the dipoles with transverse and longitudinal orientations, respectively. To show the longitudinal nature of the plasmon resonances, we also plot in Fig. 3(c) the phase of the $x$ component of the electric field induced by the dipole in the $y z$ plane close to the surface of the antenna, at two antenna resonances marked in the spectra as A and B.

Phase maps in Fig. 3(c) clearly show $l$ nodes representing the $\pi$-phase shift of the $E_{x}$ field above the nanoantenna. This analysis allows for understanding the orientation selectivity of the excited modes: by aligning the emitter in the transverse direction with respect to the antenna, we induce two symmetrically distributed regions that radiate as antiparallel dipoles yielding an even $l$ and thus no net dipole. For the longitudinal orientation, the field near the dipole is homogeneous, triggering $\pi$ shifts of the phase of 

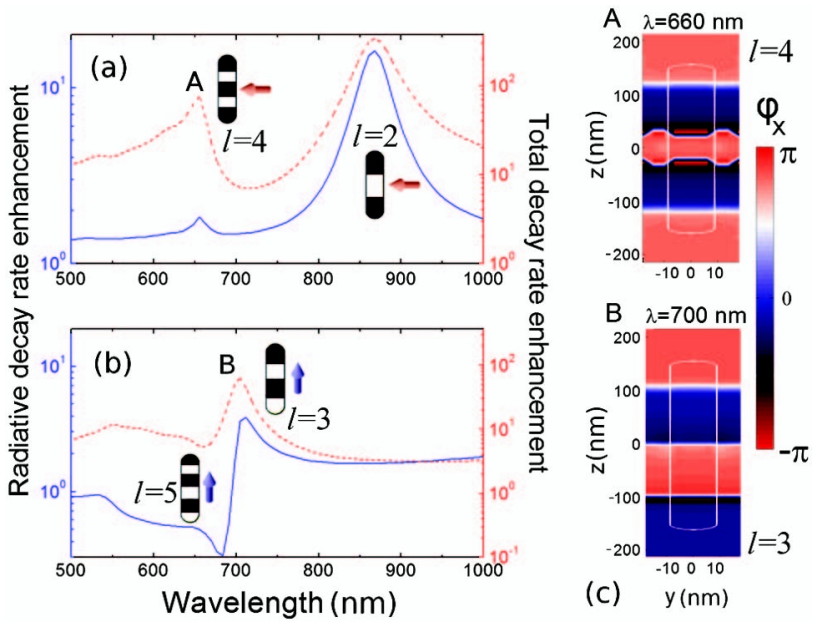

Fig. 3. Excitation of high-order plasmons in a nanoantenna by a localized emitter. Spectral dependence (a) of the enhancement of radiative (solid line) and total (dashed line) decay rate is calculated using BEM for two orientations of a dipole: transverse (upper plot) and longitudinal (lower plot). To identify these modes, a phase distribution of the electric field $E_{x}$ in the $y z$ plane close to the antenna surface is shown in (c) for two distinct modes: a dark $l=4$ mode at $\lambda=660 \mathrm{~nm}$ induced by a transversely oriented dipole (upper panel) and a bright $n=$ 3 mode excited by a longitudinally aligned dipole at $\lambda=700 \mathrm{~nm}$ (lower panel). The contour of the $20 \mathrm{~nm}$ wide nanoantenna is indicated by a solid white line.

$E_{x}$ with odd $l$ ("bright" mode). The spectral behavior of the emission for different positions and polarizations shows the potential to activate and deactivate emission rates through both bright and dark antenna modes.

In summary, we have investigated a new pathway to tune the rate of decay of a single dipolar emitter coupled to a high aspect ratio metallic antenna. We achieve this by exciting a set of spatially inhomogeneous and spectrally separated higher-order longitudinal modes in linear nanoantennas and propose an efficient and selective method for their excitation. Furthermore, we have used this notion to verify the predictions of effective wavelength theory regarding high-order modes excitation.
Consideration of the near-field behavior of nanoantennas reveals spectral features of nanorods that could not be resolved through standard far-field excitation. A close examination of the near-field coupling between elements of hybrid systems, such as the nanoantenna-localized emitter presented here, is therefore essential for a clear understanding of emission properties and might lead to new possibilities of single emitter radiation control.

Financial support from the WELCOME program "Hybrid nanostructures as a stepping-stone towards efficient artificial photosynthesis" awarded by the Foundation for Polish Science and from the ETORTEK 2011 "nanoiker" of the Department of the Industry of the Basque Country Government are gratefully acknowledged.

\section{References}

1. L. Novotny and N. van Hulst, Nat. Photon. 5, 83 (2011).

2. J. Aizpurua, G. W. Bryant, L. J. Richter, F. J. García de Abajo, B. K. Kelley, and T. Mallouk, Phys. Rev. B 71, 235420 (2005).

3. A. G. Curto, G. Volpe, T. H. Taminiau, M. P. Kreuzer, R. Quidant, and N. F. van Hulst, Science 329, 930 (2010).

4. R. Esteban, T. Teperik, and J. Greffet, Phys. Rev. Lett. 104, 026802 (2010).

5. M. Liu, T. Lee, S. K. Gray, P. Guyot-Sionnest, and M. Pelton, Phys. Rev. Lett. 102, 107401 (2009).

6. R. Artuso, G. W. Bryant, A. Garcia-Etxarri, and J. Aizpurua, Phys. Rev. B 83, 235406 (2011).

7. F. J. García de Abajo and A. Howie, Phys. Rev. B 65, 115418 (2002).

8. P. B. Johnson and R. W. Christy, Phys. Rev. B 6, 4370 (1972).

9. J.-Y. Yan, W. Zhang, S. Duan, X.-G. Zhao, and A. O. Govorov, Phys. Rev. B 77, 165301 (2008).

10. L. Novotny, Phys. Rev. Lett. 98, 266802 (2007).

11. G. Bryant, F. J. García de Abajo, and J. Aizpurua, Nano Lett. 8, 631 (2008).

12. J. Dorfmüller, R. Vogelgesang, W. Khunsin, C. Rockstuhl, C. Etrich, and K. Kern, Nano Lett. 10, 3596 (2010).

13. P. Ghenuche, S. Cherukulappurath, T. H. Taminiau, N. F. van Hulst, and R. Quidant, Phys. Rev. Lett. 101, 116805 (2008). 